\title{
Evaluation of a new LGBTQ service to complement a busy inner city GUM clinic
}

Eleanor Hamlyn, Grainne Cooney, Tristan Barber. John Hunter Clinic, Chelsea and Westminster Hospital

LGBTQ (Lesbian, Gay, Bisexual, Transgender and Queer) individuals are at significantly increased risk of sexually transmitted infections including HIV, as well as sexual violence and discrimination, substance misuse and suicide.(1-4) The need for specialist LGBTQ services in relevant health areas is increasingly recognised as an important way to facilitate individuals' to access services, with the aim of mitigating some of these risks. We decided to set up a new LGBTQ specialist clinic (reFReSH clinic) present here a service evaluation of its first eight months.

Objectives:

To evaluate a new LGBTQ service and to specifically look at:

- $\quad$ Number of patients seen and their demographics

Rates of sexually transmitted infections and to compare this to the general GUM clinic

Numbers of patients with dual diagnoses

Numbers of HIV positive individuals using the service

Methods:

Computer software was used to identify how many service users attended the service over an 8 month period and to ascertain the rates of specific STI's, existing HIV infection, number of patients accessing PEP and the number of patients seeking treatment as contacts by using the coding assigned to each patient. A month was then chosen at random to take a closer look at why individuals were using the service as well as their demographics, number of diagnoses per individual and whether they were using the service because they had had their STI diagnosed elsewhere and were then seeking treatment in a specialist clinic (i.e. after accessing online testing or Dean Street Express in Soho). This also allowed an opportunity to highlight any coding errors.

\section{Results:}

There were 31 clinics between 23rd April and 19th November with a total of 526 patient attendances for 450 individual patients. The most frequently diagnosed STI was Gonorrhoea with 60 cases, then Chlamydia with 29 cases, 24 cases of NSU, 22 cases of primary or recurrent warts, 17 cases of syphilis, 9 cases of primary or recurrent HSV. 90 patients were treated as contacts of STI's. 16 patients required PEPSE . 59 patients were HIV positive. The table opposite (Table 1) shows rates of STI's in the new service compared to the general GUM clinic over the 8 month period.

1. Increase in Sexually Transmitted Infections among Men Who Have Sex with Men, England, 2014. Public Health England, eid journal Volume 22, Number 1-January 2016

2. Psychosocial health problems increase risk for HIV among urban young men who have sex with men: Preliminary evidence of a syndemic in need of attention ; Mustanski, B et al. ann. behav. med. (2007) 34: 37. doi:10.1007/BF02879919|

3. Suicidal risk and sexual orientation in adolescence: A populationbased study in Iceland, Arnarsson et al, Scand J Public Health July 2015 vol. 43 no. 5 497-505

4. Sexual Health, HIV, and Sexually Transmitted Infections among Gay, Bisexual, and Other Men Who Have Sex with Men in the United States; Wolitski, R et al. AIDS Behav (2011) 15: 9. doi:10.1007/s10461-011-9901-6|

5. Patient Satisfaction - New LGBTQ (Lesbian Gay, Bisexual and Queer) Sexual Health Clinic. G Q (Lesbian, Gay, Bisexual and

\begin{tabular}{|l|l|l|}
\hline Table 1 & LGBTQ clinic & General GUM \\
\hline Gonorrhoea & $14.0 \%$ & $3.8 \%$ \\
\hline Chlamydia & $6.4 \%$ & $5.0 \%$ \\
\hline NSU & $5.3 \%$ & $2.9 \%$ \\
\hline Syphilis & $3.8 \%$ & $1.2 \%$ \\
\hline Warts & $4.8 \%$ & $6.3 \%$ \\
\hline HSV & $2.0 \%$ & $4.0 \%$ \\
\hline Treated as contact & $20.0 \%$ & $6.9 \%$ \\
\hline Patients requiring PEPSE & $3.5 \%$ & $1.7 \%$ \\
\hline Pts known to have HIV infection* & $13.1 \%$ & $5.7 \%$ \\
\hline
\end{tabular}

Results from the focussed 4 week period were as follows: there were 104 booked attendances 86 of whom were patients who described themselves as men who have sex with men, and 2 women who have sex with women, 9 people described themselves as bisexual, 6 as heterosexual, 1 as 'other'. The age range was $19-75$ with a mean age of 37.1. The male: female ratio was 100:4. The DNA rate was $12 \%$.

Of the 92 patients who did attend 38 had no diagnosis, 44 had one diagnosis, 9 had 2 diagnoses and one had 3 diagnoses. 24 of the patients $(26 \%)$ were HIV positive (*suggesting the figure in the above table of $13 \%$ may be an under-representation. This could be due to coding inaccuracies). 11 patients (12\%) had had an STI diagnosed elsewhere and chose to attend this clinic for treatment.

\section{Discussion:}

These high STI and HIV rates in this group, compared to the general clinic, suggest that these patients will benefit from a specialist service tailored to their needs. This involves a reconfiguration of staff compared to normal clinics to account for the increased requirements for treatments, injections and counselling. The clinic was also well received by patients and there is a qualitative assessment ${ }_{5}$, which complements this data, which demonstrates this. 